\section{Borreliose kompakt}

$\mathrm{Ni}_{\mathrm{i}}^{\mathrm{a}}$ ach sieben Jahren ist die „Klinik der Lyme-Borreliose" in 3., vollständig überarbeiteter Auflage erschienen. Neben neuen Erkenntnissen zu Immunpathologie und Labortestverfahren setzt sich Satz vor allem mit den vielfältigen Krankheitsbildern auseinander, die Haut, Bewegungsapparat, Nervensystem und Herz befallen können. Vom Erythema migrans bis zum Post-Lyme-Syndrom werden dabei auch seltene und umstrittene $\mathrm{Ma}$ nifestationen des Krankheitsspektrums behandelt.

\section{Pädiatrie für HNO-Ärzte}

$\mathrm{D}$ ie Herausgeber haben hier ein umfangreiches Werk zur pädiatrischen Otorhinolaryngologie vorgelegt wie wir es sonst nur aus der englischsprachigen Literatur kennen. Besonders gelungen sind dabei die Beiträge zur Otologie. Die entsprechenden Krankheitsbilder, Diagnostik- und Therapiemöglichkeiten betreffen einen Großteil der kindlichen HNO-Patienten und sind gut verständlich dargestellt. Unklar bleibt allerdings, warum zur akuten Otitis media und der Mastoiditis zwei eigenständige Kapitel notwendig sind. Zudem hätte man sich, gerade bei der Mastoiditis als klinisch sehr "griffigem“ Krankheitsbild mit potenziell fatalen Konsequenzen umfangreiches Bildmaterial (klinisch und radiologisch) gewünscht.
Ein Überblick über gängige Therapieempfehlungen in den verschiedenen Stadien erleichtert die Wahl der Behandlungsmethode. Auf Basis der aktuellen Studienlage werden zahlreiche Antibiotika anhand ihrer Wirksamkeit gegenübergestellt und detaillierte Therapieschemata aufgeführt. Auch kontroverse Behandlungsmethoden wie Puls- oder Phytotherapie werden anhand der entsprechenden Fachpublikationen beleuchtet.

Noch sind nicht alle klinischen Probleme zu Diagnostik, Therapie und Pro-

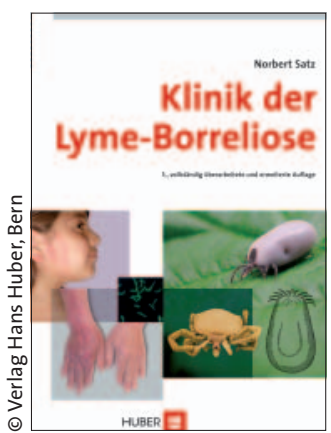

Satz N. Klinik der LymeBorreliose. Verlag Hans Huber, Bern, 2009, $629 \mathrm{~S}$. 89,95 EUR, ISBN 978-3456-84763-4

phylaxe der Lyme-Borreliose gelöst, dieses Buch fasst aber schon jetzt den aktuellen Stand des Wissens zusammen und weist auf bestehende Lücken hin.

red

Auch bei den Komplikationen der $\mathrm{Na}$ sennebenhöhlenentzündungen wäre neben einer ausführlicheren Beschreibung der Krankheitsbilder anschauliches Bildmaterial sinnvoll gewesen - schließlich handelt es sich hier um Komplikationen, deren Nichterkennen oder Fehleinschätzung ernsthafte Folgen nach sich ziehen kann. Die Abbildungen, von denen ein Lehrbuch natürlich ebenfalls lebt, sind in der Mehrzahl sehr anschaulich. Ärgerlich wird es allerdings, wenn der Rezensent Bildmaterial (von Erwachsenen und ohne Quellenangabe!) aus seinen eigenen Lehrbüchern wieder erkennt, die als „kindliche" Befunde ausgegeben werden.

Diese Beispiele sollen die Qualität des weitgehend sehr gelungenen und lehrreichen Gesamtwerkes keineswegs infrage

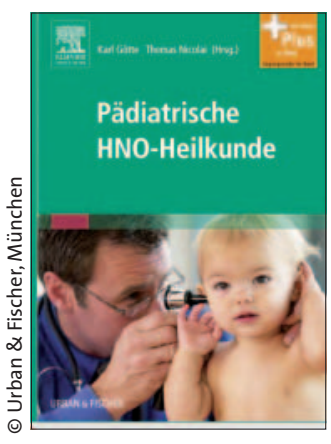

Götte N \& Nicolai T. Pädiatrische HNO-Heilkunde. Urban \& Fischer, München, 2009, 684 S., 129 EUR, ISBN 978-3437-24660-9

stellen, gewisse Schwachstellen lassen sich in einem Multiautorenbuch selten vermeiden. Zusammengefasst ist das Buch eine sinnvolle Ergänzung der aktuellen otorhinolaryngologischen Literatur und gehört deshalb auch in die Bibliothek eines jeden HNO-Arztes, der für sich in Anspruch nimmt, nach zeitgemäßen Richtlinien zu behandeln.

gg

\title{
Ein volles Pfund Praxiswissen
}

$\mathrm{N}$ eun Jahre ist es her, dass die „Praxis der HNO-Heilkunde" erstmals erschienen ist. Die 2. Auflage ist wie der Vorgänger aufgebaut: Nach einem ausführlichen Kapitel zur Diagnostik werden die einzelnen Organe unseres Fachgebietes sukzessive aufgeführt. Im Rahmen der „speziellen chirurgischen Therapie“ schließlich werden operative Verfahren systematisch und reich bebildert vorgestellt. Hier finden sich entsprechend Unterkapitel zur „Plastischen und rekonstruktiven Chirurgie“, "Chirurgischen Therapie der Orbita", Laserchir- urgie und Schlafmedizin. Warum auch ein Kapitel über Psychosomatik unter der „speziellen chirurgischen Therapie“ erscheint, bleibt allerdings unverständlich, hier hätte man sinnvollerweise ein separates Kapitel analog zur Chirotherapie anfügen können. Eigene Kapitel zur konservativen Therapie, Notfallbehandlung und Phoniatrie sowie Hörgeräteversorgung runden das insgesamt gelungene Bild des Gesamtwerkes ab. Wer tiefer einsteigen will, findet ein übersichtliches und aktualisiertes Literaturverzeichnis am Ende eines jeden Kapitels.

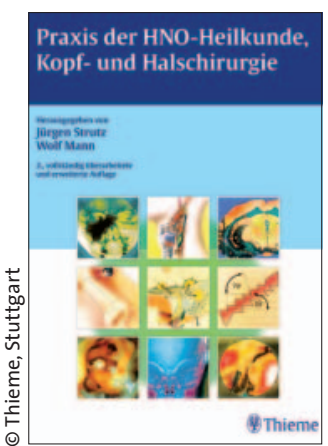

Bezüglich Umfang (fast 1.100 Seiten) und Inhalt gibt es gegenwärtig sicher kein vergleichbares Werk zur HNOHeilkunde in deutscher Sprache.

\section{Strutz J \& Mann W. Praxis der HNO-Heilkun- de, Kopf- und Halschirurgie. Georg Thieme Verlag, Stuttg- art, 2009, 1096 S., 249,95 EUR, ISBN 978-3-131- 16972-3}

.

